\title{
Altered perfusion of the sensorimotor cortex in patients with cervical spondylotic myelopathy: an arterial spin labeling study
}

This article was published in the following Dove Press journal: Journal of Pain Research

\author{
Fuqing Zhou',2 \\ Muhua Huang ${ }^{1,2}$ \\ Lin $\mathrm{Wu}^{1,2}$ \\ Yongming $\operatorname{Tan}^{1,2}$ \\ Jianqiang Guo ${ }^{1,2}$ \\ Yong Zhang ${ }^{3}$ \\ Laichang $\mathrm{He}^{1,2}$ \\ Honghan Gong ${ }^{1,2}$ \\ 'Department of Radiology, the \\ First Affiliated Hospital, Nanchang \\ University, Nanchang, Jiangxi \\ Province, People's Republic of China; \\ ${ }^{2}$ Jiangxi Province Medical Imaging \\ Research Institute, Nanchang, Jiangxi \\ Province, People's Republic of China; \\ ${ }^{3}$ Department of Pain Clinic, the First \\ Affiliated Hospital of Nanchang \\ University, Nanchang, Jiangxi Province, \\ People's Republic of China
}

Correspondence: Laichang He Department of Radiology, The First Affiliated Hospital, Nanchang University, 17 Yongwaizheng Street, Nanchang 330006, Jiangxi Province, People's

Republic of China

Tel +86 79l 88692773

Email laichang_he@I63.com

Yong Zhang

Department of Pain Clinic, The First

Affiliated Hospital, Nanchang University,

17 Yongwaizheng Street, Nanchang

330006, Jiangxi Province, People's

Republic of China

Tel +8679l 88692773

Email zy830226@I63.com
Objective: Advanced magnetic resonance imaging studies have shown functional plasticity or reorganization and metabolite alterations of $\mathrm{N}$-acetyl aspartate in the sensorimotor cortex (SMC), a hallmark region and key brain network, in patients with cervical spondylotic myelopathy (CSM). However, the nature of perfusion in the SMC and the relationship between regional cerebral blood flow (CBF), motor function scores, and structural damage of the cervical cord in patients with CSM are not fully understood.

Materials and methods: All right-handed participants underwent pseudo-continuous arterial spin labeling pulse sequence scanning, and CBF was then calculated and compared between CSM and healthy groups. Clinical and structural associations were assessed in the SMC. Receiver operating characteristic (ROC) and leave-one-out cross-validation analyses were used to estimate the sensitivity and specificity of the significantly altered CBF in the SMC to distinguish myelopathy-related impairment.

Results: A total of 18 pairs of CSM patients and well-matched healthy subjects were included in the analyses. Compared with healthy subjects, CSM patients exhibited significantly decreased $\mathrm{CBF}$ in the left premotor ventral/precentral operculum $(\mathrm{PMv} / \mathrm{PrCO})$ and the bilateral dorsal anterior cingulate cortex (dACC); and increased $\mathrm{CBF}$ in the left paracentral lobule (PCL), the right $\mathrm{PCL} /$ supplementary motor area (PCL/SMA), and the right postcentral gyrus (PoCG; Gaussian random field correction at $P<0.01$ ). In the CSM group, the CBF values in the right PoCG were negatively correlated with Japanese Orthopaedic Association scores, and the CBF values in several regions were negatively correlated with Neck Disability Index scores. Finally, the ROC analysis revealed that significantly increased CBF in the left PCL, the right PCL/SMA, and the right PoCG discriminated patients with myelopathy-related impairment from healthy subjects. Conclusion: Regional $\mathrm{CBF}$ was reduced in operculum-integrated (PMv/PrCO) and motor control (dACC) regions but increased in sensory (PoCG) and motor-sensory processing (PCL/ SMA) regions in patients with CSM.

Keywords: regional cerebral blood flow, cervical spondylotic myelopathy, arterial spin labeling

\section{Introduction}

Cervical spondylotic myelopathy (CSM) is a common spinal cord disorder that causes neck pain and motor and sensory deficits of the limbs in middle-aged people. The main clinical symptoms of CSM are caused by local injury to the ascending or descending spinal cord fibers due to incomplete compression of the spinal cord or by nerve root entrapment. ${ }^{1-4}$ However, evidence from recent neuroimaging studies has suggested that cortical plasticity or reorganization may also affect the clinical symptoms, manifestations, and rehabilitation of CSM patients. ${ }^{1,5}$ 
CSM patients exhibit functional abnormalities of the central nervous system, including increased task-related activation in the primary motor and premotor cortices; ${ }^{6-8}$ loss of activation in the sensory cortex $;^{8}$ decreased intrinsic connectivity in the operculum-integrated regions; and increased connectivity in the premotor, right $\mathrm{S} 1$, and parietal-integrated regions. ${ }^{1}$ Moreover, post-surgical central functional adaptations or recruitment accompany the improvement of clinical symptoms or functional recovery in CSM patients. ${ }^{6-9}$ In addition, studies have also reported metabolite alterations of $\mathrm{N}$-acetyl aspartate in the motor cortex in pre- ${ }^{10}$ and postsurgery ${ }^{5}$ CSM patients. ${ }^{11}$ These findings provide a better theoretical basis for understanding the cortical plasticity or reorganization in CSM patients.

In our previous studies, we demonstrated alterations in intrinsic oscillation amplitudes ${ }^{12}$ and local ${ }^{13}$ and longdistance $^{14}$ connectivity in the sensorimotor cortex (SMC), a hallmark region and key brain network, in patients with CSM. However, the physiological mechanisms underlying the intrinsic functional plasticity of the SMC in CSM patients are poorly understood. Recent studies have observed tight coupling between regional cerebral blood flow (CBF) and intrinsic connectivity strength during both resting and taskactivated brain states. ${ }^{15,16}$ However, only a few studies have used ${ }^{18} \mathrm{~F}$-FDG PET to observe perfusion around the cervical cord in CSM patients, ${ }^{17,18}$ and relatively little is known about SMC perfusion using non-invasive arterial spin labeling (ASL) in CSM patients.

The goal of this study was to investigate alterations of perfusion in the SMC using a pseudo-continuous ASL (pCASL) pulse sequence. pCASL is a promising imaging technique used to visualize $\mathrm{CBF}$ with lower inter-subject variability. ${ }^{19}$ pCASL can be applied to study various vascular diseases, such as stroke ${ }^{20}$ and "functional" diseases, including Alzheimer's disease ${ }^{21}$ and schizophrenia. ${ }^{22}$ In this study, we hypothesized that alterations in CBF would exist in the SMC of patients with CSM and that these alterations would be related to motor function, as measured by Japanese Orthopaedic Association (JOA) scores or severity of structural damage to the cervical cord.

\section{Materials and methods Ethical statement}

The current study was approved by the Medical Research Ethics Committee and the Institutional Review Board of the First Affiliated Hospital, Nanchang University, People's Republic of China. This study was also conducted according to approved guidelines and in compliance with the principles of the Declaration of Helsinki. Before magnetic resonance scanning, written informed consent was obtained from each participant.

\section{Participant and clinical estimates}

Forty-two right-handed participants were recruited from a local hospital and the urban community and comprised 21 pre-surgery CSM patients and 21 age-, gender-, and education-matched healthy control (HC) subjects. The CSM group included a consecutive series of patients who met the study inclusion/exclusion criteria. Some patients had participated in previous resting-state functional magnetic resonance imaging (fMRI) studies, ${ }^{1,12-14}$ and detailed descriptions of the inclusion criteria can be found in previous reports. ${ }^{1}$ Two radiologists assessed the spinal cord compression, defined as a clearly indented cord surface or by a narrowed cord diameter with hyper-intensity of the cord on T2-weighted images, which indicated that these CSM patients had intrinsic T2 changes.

The severity of cervical myelopathy was evaluated by the JOA system and Neck Disability Index (NDI) questionnaires. The JOA system evaluates the clinical severity of myelopathy by assigning scores based on the degree of dysfunction, and the NDI was designed to measure the activities of daily living in patients with neck pain.

For imaging data quality control, participants were excluded if the maximum displacement in any orthogonal direction ( $\mathrm{x}, \mathrm{y}, \mathrm{z}$ ) exceeded $2 \mathrm{~mm}$ or if angular head motion exceeded $2.0^{\circ}$ at any time during the pCASL scan. In this study, two patients and one healthy subject had head movement $>2 \mathrm{~mm}$ or $>2.0^{\circ}$ during the pCASL scan and were excluded. Considering the paired data, the associated paired patient and healthy subjects were also excluded from the study. Finally, a total of 18 paired patients and healthy subjects were included in the present study. The detailed demographic and clinical data of all subjects are shown in Table 1 .

\section{MRI data acquisition}

MRI data acquisition was conducted using a 3.0-T MR system (Trio; Siemens, Munich, Germany) at the First Affiliated Hospital. Each participant underwent a resting pCASL sequence scan with eyes closed and high-resolution 3-dimensional (3D) T1-weighted image (T1WI) acquisition with foam padding in an 8-channel phased-array head coil for restricted head motion. 1) pCASL was used to acquire perfusion data with the following protocol: repetition time (TR), $4000 \mathrm{~ms}$; echo time (TE), $12 \mathrm{~ms}$; time interval between consecutive slice acquisitions, $32.0 \mathrm{~ms}$; radio frequency duration, 0.5 
Table I Demographic and clinical information for the CSM and HC groups (mean \pm SD)

\begin{tabular}{llll}
\hline Clinical information & CSM & HC & $t(P)$-values \\
\hline $\mathrm{n}$ & 18 & 18 & $\mathrm{~N} / \mathrm{A}$ \\
Age, years & $51.94 \pm 7.8 \mathrm{I}$ & $50.50 \pm 7.12$ & $0.580(0.879)$ \\
Gender (male/female) & $13 / 5$ & $13 / 5$ & $\mathrm{~N} / \mathrm{A}$ \\
Education (years) & $14.06 \pm 1.16$ & $14.17 \pm 1.21$ & $-2.556(0.801)$ \\
Duration of symptoms (months) & $10.11 \pm 10.04$ & $0.00 \pm 00$ & $\mathrm{~N} / \mathrm{A}$ \\
NDI scores & $31.20 \% \pm 11.01 \%$ & $2.89 \% \pm 4.76 \%$ & $10.014(0.0001)$ \\
JOA scores & $11.67 \pm 2.74$ & $17.00 \pm 0.00$ & $-8.246(0.0001)$ \\
Motor upper & $2.17 \pm 0.78$ & $4.00 \pm 0.00$ & $-9.897(0.0001)$ \\
Motor lower & $3.22 \pm 1.16$ & $4.00 \pm 0.00$ & $-2.830(0.008)$ \\
Sensor deficit & $3.33 \pm 0.84$ & $6.00 \pm 0.00$ & $-13.46(0.000 I)$ \\
Bladder dysfunction & $2.94 \pm 0.23$ & $3.00 \pm 0.00$ & $-1.000(0.324)$ \\
FA values at the C2 level & $0.59 \pm 0.048$ & $0.66 \pm 0.042$ & $-4.892(0.000 I)$
\end{tabular}

Abbreviations: CSM, cervical spondylotic myelopathy; FA, fractional anisotropy; HC, healthy control; JOA, Japanese Orthopaedic Association; N/A, not applicable; NDI, Neck Disability Index.

ms; labeling duration $(\tau), 1650 \mathrm{~ms}$; labeling pulse flip angle, $18^{\circ}$; post-spin labeling delay $(\omega), 1520 \mathrm{~ms}$; bandwidth, 3.3 $\mathrm{kHz} /$ pixel; field of view (FOV), $240 \times 240$; matrix, $64 \times 64$; $3.44 \times 3.44 \times 4 \mathrm{~mm}^{3}$ resolution; 20 axial slices, $4 \mathrm{~mm}$ slice thickness with $1 \mathrm{~mm}$ gap; and background suppression. The labeling block was $90 \mathrm{~mm}$ below the isocenter of the readout slices (tenth slice of scanning), 80 label/control image pairs were acquired and total scan duration of $320 \mathrm{~s}$ for each participant. 2) Sagittal 3D T1WI were acquired with the following protocol for ASL data processing: TR/TE, 2,530 $\mathrm{ms} / 3.39 \mathrm{~ms}$; flip angle, $7^{\circ}$; thickness, $1.33 \mathrm{~mm}$; gap, $0 \mathrm{~mm}$; matrix, $256 \times 256$; FOV, $240 \mathrm{~mm} \times 240 \mathrm{~mm}$, and a total of 176 contiguous slices through the brain. 3 ) Axial diffusion tensor imaging covering the cervical spinal cord from $\mathrm{C} 1$ to C7 was acquired to evaluate cervical cord micro-structural damage, and the spin echo single-shot echo planar sequence parameters were as follows: $\mathrm{TR} / \mathrm{TE}=5000 / 106 \mathrm{~ms}$; FOV $=$ $128 \times 124 \mathrm{~mm} ; \mathrm{NEX}=2$; matrix $=128 \times 124$; slice thickness $=5 \mathrm{~mm} ; 20$ nonlinear diffusion-weighted gradient directions with $\mathrm{b}=600 \mathrm{~s} / \mathrm{mm}^{2}$ and one additional image without diffusion weighting (i.e., $b=0 \mathrm{~s} / \mathrm{mm}^{2}$ ). 3 ) In addition, we also acquired sagittal and axial conventional T1-weighted, T2-weighted and T2-fluid-attenuated inversion recovery (FLAIR) images of the brain and cervical spinal cord for diagnostic purposes.

\section{pCASL processing and analyses}

The pCASL and high-resolution T1WI data were processed using the ASLtbx toolbox (https:/www.cfn. upenn.edu/ ASLtbx.php) in MATLAB (Math Works, Natick, MA, USA). Data processing was implemented as follows: 1) perprocessing: median filter (nonlinear digital filtering technique) for reducing spike noise; 2) head motion correction: participants who had greater than $2^{\circ}$ of angular rotation along any axis or greater than $2 \mathrm{~mm}$ of translation along any axis during the functional MR scanning were rejected; 3) data segment, co-registration and spatial normalization: high-resolution individual T1-anatomic images were registered and then segmented and transformed into the standard Montreal Neurological Institute $\left(2 \times 2 \times 2 \mathrm{~mm}^{3}\right)$ space using the DARTEL toolbox,${ }^{23}$ and the resulting normalization parameters were used to normalize the pCASL data; 4) smoothing: a $5 \mathrm{~mm}$ full-width at half maximum Gaussian kernel was applied to decrease spatial noise and to compensate for imperfect normalization. The model for quantifying $\mathrm{CBF}$ is given by equation below , and the model was implemented in ASLtbx:

$$
C B F=\frac{\Delta M \lambda R_{1 a} \exp \left(\omega R_{1 a}\right)}{2 M_{0} a}\left[1-\exp \left(-\tau R_{1 a}\right)\right]^{-1}
$$

where $\Delta \mathrm{M}$ is the difference between the control and label values, $\lambda$ is blood/tissue water partition coefficient $(0.9 \mathrm{~mL} / \mathrm{g})$, $R_{1 a}$ is the longitudinal relaxation rate of blood (1600 ms), $\tau$ is the labeling time, $\alpha$ is the labeling efficiency (0.95), $\omega$ is the post-labeling delay time, and $\mathrm{M}_{0}$ is the equilibrium magnetization of the brain.

\section{Fractional anisotropy (FA) metrics calculation in the cervical cord}

FA metrics of the $\mathrm{C} 2$ vertebral level and the most severe compression level were calculated for the diffusion tensor imaging native space for patients with CSM using the Diffusion Toolkit (http://www.trackvis.org/) to evaluate the microstructural severity of the cervical canal stenosis. 


\section{Statistical analyses}

In the SMC, group differences of ASL perfusion between the CSM and HC groups were tested by voxel-wise, two sample $t$-tests with age and gender as covariates (Statistical Parametric Mapping 12, http://www.fil.ion.ucl.ac.uk/spm). Multiple comparison corrections were performed using the Gaussian random field (GRF) theory with a voxel level of $P<0.01$ and a cluster level of $P<0.05$. Furthermore, we correlated the regional $\mathrm{CBF}(\mathrm{rCBF})$ values within the SMC with Fisher $Z$-scores in each patient to determine whether the reduced perfusion in patients with CSM was related to the micro-structural severity (FA values at $\mathrm{C} 2$ and most severe compression levels), clinical severity (JOA scores) of cervical myelopathy, activities of daily living (NDI), or disease duration $(P<0.01$, GRF-corrected in cluster level at $P<0.05)$.

\section{Discriminant analysis}

In the discriminant analysis of the altered pCASL within the SMC, receiver operator characteristic (ROC) curves were used to estimate the sensitivity and specificity for distinguishing CSM patients from healthy subjects. The area under the ROC curve was used to estimate the accuracy of the classification. A leave-one-out cross-validation (LOOCV) was used to assess the validity of the results.

\section{Results}

\section{Demographic and clinical data}

In this study, we obtained 18 paired CSM patients and HC subjects for analysis. Among these CSM patients, 38.9\% had single-level compression and $61.1 \%$ had two or more levels of compression secondary to disk degeneration, spondylosis, or both. A total of $72.2 \%$ presented with pain or discomfort in the neck or shoulder; $61.1 \%$ presented with weakness, a loss of dexterity, or sensory loss in the upper limbs; and 55.6\% had gait instability. The demographic and clinical data of the study groups are shown in Table 1 .
There were no significant differences between the groups with respect to age $(P=0.879)$ or gender. In this study, all the healthy subjects scored maximum values on all sub-sections of the JOA test, resulting in the maximum score of 17, but CSM patients had significantly lower JOA scores (11.67 \pm 2.74, range: $3-14)$. In comparison to the HC group, CSM patients had significantly higher NDI scores $(31.20 \% \pm$ $11.01 \%$ vs. $2.89 \% \pm 4.76 \%$ ) and lower FA values at the $\mathrm{C} 2$ level $(0.59 \pm 0.048$ vs. $0.66 \pm 0.042)$. The mentioned clinical measures revealed disabilities and loss of integrity due to cervical myelopathy. A total of 18 patients received surgical decompression to relieve their clinical symptoms at the First Affiliated Hospital, Nanchang University.

\section{CBF difference between groups}

The spatial patterns of CBF across the whole brain for the $\mathrm{HC}$ group and CSM patients are shown in Figure 1. In the whole brain, high $\mathrm{CBF}$ was observed in the precuneus and posterior cingulate regions, the anterior cingulate cortex (ACC) and middle prefrontal cortex, the precentral gyrus (PrCG), and the postcentral gyrus (PoCG). In this study, group comparisons were focused on the SMC, primarily the bilateral PrCG, the PoCG, the supplementary motor area (SMA), the inferior frontal gyrus and a portion of the middle cingulate cortex, the paracentral lobule (PCL), and part of the bilateral insula. Compared with $\mathrm{HC}$, the CSM patients showed lower CBF in the left premotor ventral/precentral operculum ( $\mathrm{PMv} / \mathrm{PrCO})$ and bilateral dorsal ACC (dACC), but higher CBF in the left PCL, the right PCL/ SMA, and the right PoCG, as shown in Figure 2 and Table 2.

The results were mapped on cortical surfaces using BrainNet viewer (www.nitrc.org/projects/bnv).

\section{Correlations between rCBF values and clinical data in CSM patients}

In CSM patients, significant negative correlations were found between the rCBF values and the JOA scores in the

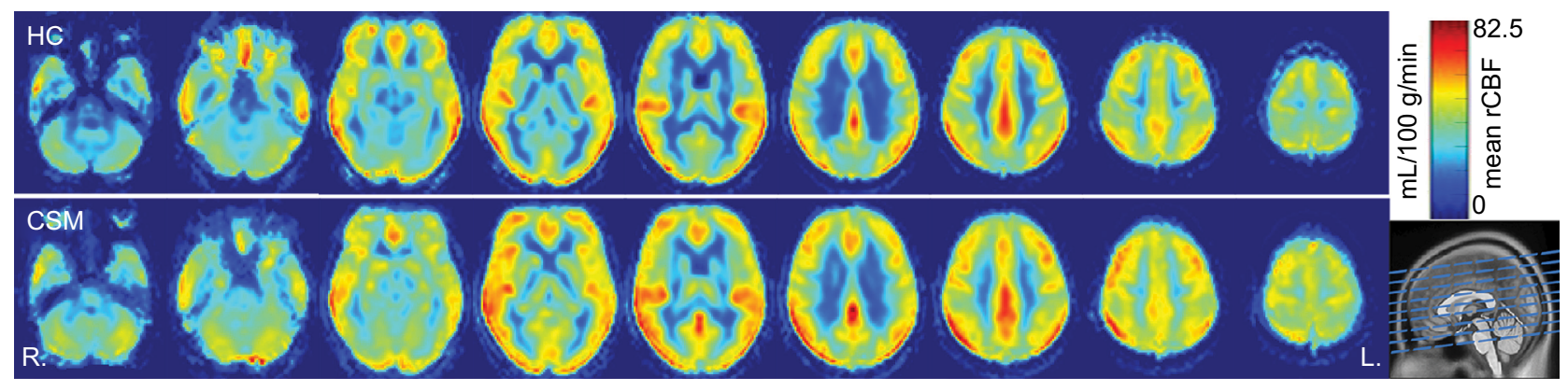

Figure I Maps of CBF of $\mathrm{HC}$ subjects ( $\mathrm{n}=18$, upper row) and patients with CSM ( $\mathrm{n}=18$, lower row). Abbreviations: CBF, cerebral blood flow; CSM, cervical spondylotic myelopathy; HC, healthy control. 


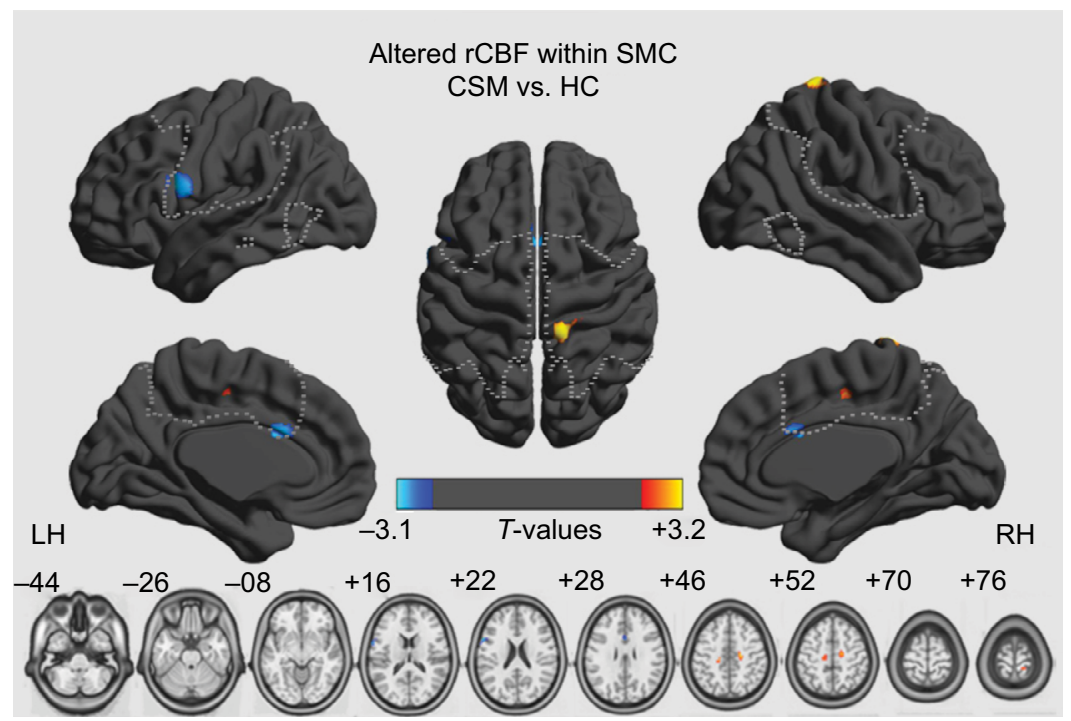

Figure 2 Significant rCBF differences between healthy control subjects and patients with cervical spondylotic myelopathy within the sensorimotor cortex (two sample $t$-tests, $P<0.0$ I, GRF-corrected in cluster level at $P<0.05$; yellow-red and cyan-blue colors indicate higher and lower perfusion in the patients, respectively).

Abbreviations: CSM, cervical spondylotic myelopathy; GRF, Gaussian random field; HC, healthy control; rCBF, regional cerebral blood flow; SMC, sensorimotor cortex.

Table 2 The CSM group showed altered CBF within the sensorimotor cortex (two sample $t$-tests, $P<0.0$ I, GRF-corrected at the cluster level at $P<0.05)$

\begin{tabular}{llllll}
\hline $\begin{array}{l}\text { Cluster size } \\
\text { (voxels) }\end{array}$ & Peak T-score & \multicolumn{2}{l}{ Peak MNI coordinates } & Brain region \\
\cline { 2 - 5 } & & $\mathbf{x}$ & $\mathbf{y}$ & $\mathbf{z}$ & \\
\hline 60 & -3.07 & -60 & 12 & 20 & Left premotor ventral/precentral operculum \\
24 & -2.89 & -2 & 14 & 26 & Bilateral dorsal anterior cingulate cortex \\
77 & 2.87 & -20 & -26 & 44 & Left paracentral lobule \\
118 & 3.11 & 18 & -16 & 48 & Right paracentral lobule/ supplementary motor area \\
29 & 3.16 & 14 & -38 & 78 & Right postcentral gyrus \\
\hline
\end{tabular}

Abbreviations: CBF, cerebral blood flow; CSM, cervical spondylotic myelopathy; GRF, Gaussian random field; MNI, Montreal Neurological Institute.

right PoCG (Table 3 and Figure 3A) and between the rCBF values and the NDI scores in the left $\mathrm{PMv} / \mathrm{PrCO}$, the right $\mathrm{PMv} / \mathrm{PrCO}$, the left supramarginal gyrus, the bilateral SMA, the right PoCG, the left middle cingulum cortex, and the right dorsolateral premotor cortex ( $\mathrm{PMd}$; Table 4 and Figure 3B; $P<0.01$, GRF-corrected in cluster level at $P<0.05)$. No significant relationship was observed between $\mathrm{rCBF}$ values and disease duration, or between $\mathrm{rCBF}$ values and $\mathrm{FA}$ values at the $\mathrm{C} 2$ or the most severe compression level.

\section{Discriminatory performance analysis of the $\mathrm{rCBF}$}

Some regions exhibited significant between-group differences in CBF. Higher CBF in the left PCL, the right PCL/ SMA, and the right PoCG may be able to be used to discriminate between CSM patients and HC. To investigate this possibility, the mean $\mathrm{CBF}$ values in the three regions were extracted. ROC analysis revealed that the areas under the curves (AUCs) were 0.738 for the left PCL, 0.754 for the right PCL/SMA, and 0.590 for the right PoCG (Figure 4 and Table S1). The LOOCV showed cross-validated AUCs of $0.738 \pm 0.0101(0.725-0.760)$ for the left PCL, $0.754 \pm$ $0.0158(0.738-0.792)$ for the left right PCL/SMA, and 0.590 $\pm 0.0183(0.563-0.625)$ for the right PoCG.

\section{Discussion}

In this study, CBF was altered in the SMC of patients with CSM compared to $\mathrm{HC}$ subjects. The CBF measures were lower in CSM patients than in controls in the left PMv/ PrCO and in the bilateral dACC, indicative of regional hypoperfusion or dysfunction. CSM patients had higher CBF than controls in the left PCL, the right PCL/SMA, and the right PoCG. Our data suggest that there are alterations of perfusion in the SMC in patients with CSM.

In the CSM group, decreased $\mathrm{CBF}$ was observed in the left $\mathrm{PMv} / \mathrm{PrCO}$ and the right $\mathrm{dACC}$, which was negatively correlated with the NDI score. Previous anatomical and functional connectivity studies have revealed that the $\mathrm{PMv} / \mathrm{PrCO}$ 
Table 3 Regions with significant correlations between JOA scores and rCBF in the CSM group $(P<0.0$ I, GRF-corrected at the cluster level at $P<0.05$ )

\begin{tabular}{|c|c|c|c|c|c|}
\hline \multirow{2}{*}{$\begin{array}{l}\text { Cluster size } \\
\text { (voxels) }\end{array}$} & \multirow[t]{2}{*}{ Peak r-score } & \multicolumn{3}{|c|}{ Peak MNI coordinates } & \multirow[t]{2}{*}{ Brain region } \\
\hline & & $\mathbf{x}$ & $y$ & $\mathbf{z}$ & \\
\hline 30 & -0.683 & 50 & -28 & 60 & Right postcentral gyrus \\
\hline
\end{tabular}

Abbreviations: CSM, cervical spondylotic myelopathy; GRF, Gaussian random field; JOA, Japanese Orthopaedic Association; MNI, Montreal Neurological Institute; rCBF, regional cerebral blood flow.

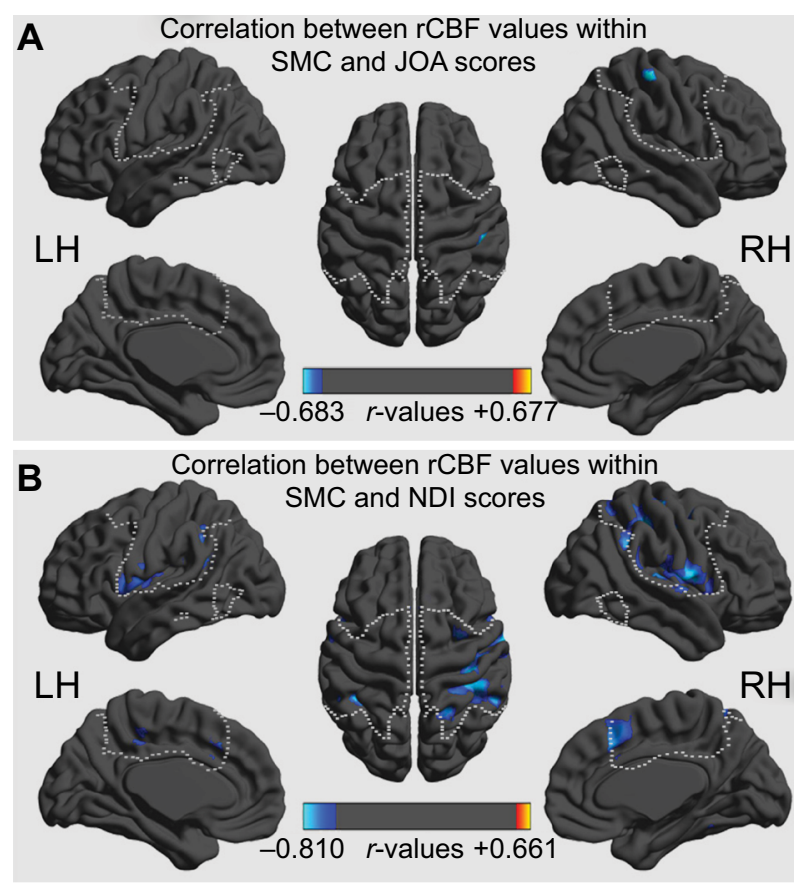

Figure 3 Correlations of $\mathrm{rCBF}$ values with JOA scores $(\mathbf{A})$ and NDI scores $(\mathbf{B})$ in CSM patients $(P<0.0$ I, GRF-corrected at the cluster level at $P<0.05)$.

Abbreviations: CSM, cervical spondylotic myelopathy; GRF, Gaussian random field; JOA, Japanese Orthopaedic Association; NDI, Neck Disability Index; rCBF, regional cerebral blood flow; SMC, sensorimotor cortex.

is a key node in the network for integrative processes. ${ }^{24}$ The $\mathrm{PMv} / \mathrm{PrCO}$ receives input information involved in locating one's own limb, subjective limb ownership, and polymodal action recognition. ${ }^{25-27}$ In this study, decreased CBF of the right $\mathrm{PMv} / \mathrm{PrCO}$ was also significantly correlated with NDI scores, which indicates a perfusion-related, self-rated disability. Patients with CSM frequently experience tingling or numbness in the arms, fingers or hands, resulting in loss of fine motor skills. Decreased activity of the PMv/PrCO was detected in a previous study of myelopathy patients, ${ }^{1}$ and a similar perception loss also occurs in the "rubber hand illusion". ${ }^{25}$ The decreased CBF of the PMv/PrCO found in this study may help us understand the extent to which CSM patients with neck pain (high NDI) face disability in daily living activities.

The dACC is a key brain region involved in motor control $^{28,29}$ and cognition, ${ }^{30}$ and selectively modulates the
SMA during coordinated motor behaviors or motor task conditions. ${ }^{28,29}$ Previous studies demonstrated that the dACC connectivity network is significantly associated with motor function and cognitive function, both during tasks and resting states. ${ }^{28,29,31}$ Bidirectional interactions exist between the dACC and the SMA. Bottom-up connectivity (from the SMA to the $\mathrm{dACC}$ ) is specific to motor coordination during resting state, but top-down connectivity (from the dACC to the SMA) is exerted during book-ended tasks. ${ }^{29}$ In CSM patients, one type of "myelopathy hand" is characterized by localized wasting and weakness of the intrinsic and extrinsic hand muscles without sensory loss or spastic quadriparesis. In our study, although no significant relationship was detected between the CBF of the dACC and JOA scores, decreased CBF of the right $\mathrm{dACC}$ was negatively correlated with NDI scores. In particular, in patients with spinal cord injury, the reduced regional blood flow in cerebral motor and sensory areas indicates dysfunctional $\mathrm{CBF}$ regulation of brain function. ${ }^{32}$ Given the existing literature,${ }^{5}$ we believe that dysfunction of the dACC due to hypo-perfusion may contribute to the clinical presentation of CSM by the motor sub-network.

We also noted that the CSM group showed significantly increased $\mathrm{CBF}$ in the left PCL, the right PCL/SMA, and the right PoCG. In addition, several regions, including the PoCG, exhibited a significant correlation between the increased rCBF and JOA scores or NDI scores (Figure 4). NDI was designed for measurements of "myelopathy daily living" but JOA for "myelopathy dysfunction". In fact, the PoCG, as the main sensory receptive area, is one of the major regions affected in CSM patients, ${ }^{33}$ while the PCL controls motor and sensory innervations. ${ }^{34}$ In a resting-state fMRI study of CSM, one explanation of increased connectivity is a negative "side effect" in the context of lost sensory impulses. ${ }^{1,6}$ Hyperperfusion in the aforementioned regions further supports the "adaptive plasticity" hypothesis posed by Zhou et al. ${ }^{1}$ derived from the blood flow profile. ${ }^{11,18}$ Another explanation is that a loss of afferent sensory conditions may result in less efficient funneling of neural processing. ${ }^{34}$

A previous study demonstrated that metabolic, structural, and functional abnormalities of the central nervous system 
Table 4 Regions with significant correlations between NDI scores and rCBF in the CSM group $(P<0.0$ I, GRF-corrected at the cluster level at $P<0.05$ )

\begin{tabular}{|c|c|c|c|c|c|}
\hline \multirow{2}{*}{$\begin{array}{l}\text { Cluster size } \\
\text { (voxels) }\end{array}$} & \multirow{2}{*}{$\begin{array}{l}\text { Peak } \\
\text { r-score }\end{array}$} & \multicolumn{3}{|c|}{ Peak MNI coordinates } & \multirow[t]{2}{*}{ Brain region } \\
\hline & & $\mathbf{x}$ & $\mathbf{y}$ & $\mathbf{z}$ & \\
\hline 445 & -0.759 & -38 & -22 & 28 & Left premotor ventral/precentral operculum \\
\hline 849 & -0.785 & 60 & -2 & 12 & Right premotor ventral/precentral operculum \\
\hline 344 & -0.811 & -46 & -46 & 34 & Left supramarginal gyrus \\
\hline 246 & -0.736 & 2 & 22 & 42 & Bilateral supplementary motor area \\
\hline 861 & -0.803 & 40 & -26 & 62 & Right postcentral gyrus \\
\hline 49 & -0.673 & -6 & -32 & 46 & Left dorsal anterior cingulate \\
\hline 42 & -0.716 & 32 & 0 & 56 & Right dorsolateral premotor cortex \\
\hline
\end{tabular}

Abbreviations: CSM, cervical spondylotic myelopathy; GRF, Gaussian random field; MNI, Montreal Neurological Institute; NDI, Neck Disability Index; rCBF, regional cerebral blood flow.

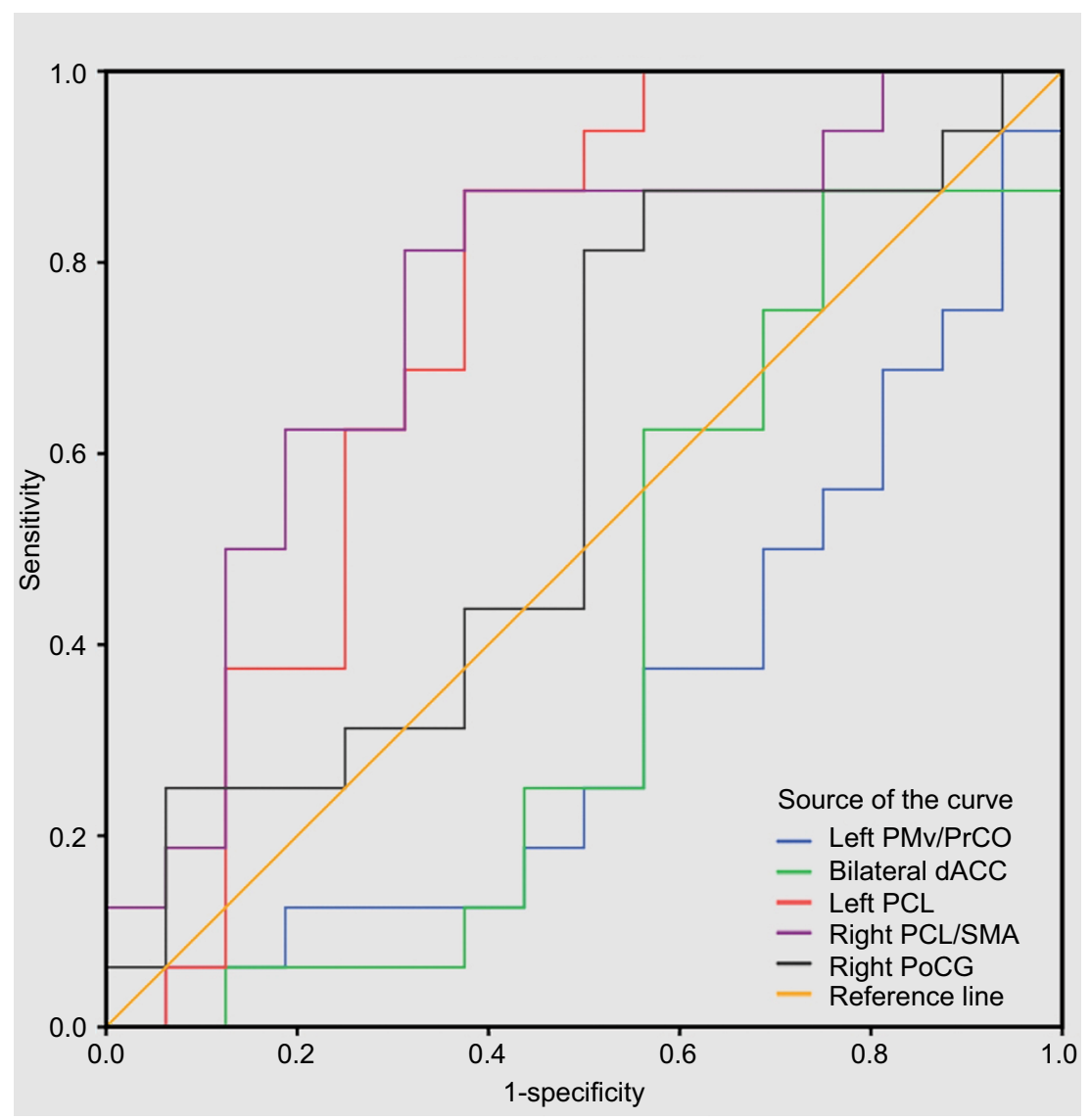

Figure 4 ROC analysis for differentiating myelopathy-related impairment between SMC patients and healthy subjects.

Abbreviations: $\mathrm{dACC}$, dorsal anterior cingulate cortex; PCL, paracentral lobule; PMv/PrCO, premotor ventral/precentral operculum; PoCG, postcentral gyrus; ROC, receiver operating characteristic; SMA, supplementary motor area; SMC, sensorimotor cortex.

of CSM patients, ${ }^{6-8}$ including a decreased $N$-acetyl aspartate/ creatine metabolite ratio, ${ }^{5,35}$ increased task-related activation and alterations in intrinsic activity, and are indicative of neuronal damage or dysfunction in the SMC. The current findings add to previous studies of neuroimaging suggesting myelopathy-related alterations of neural intrinsic activity in the $\mathrm{SMC}^{1,12}$ and extend these findings into the perfusion domain. Whether these findings are related to intrinsic neural activity (i.e., amplitude of low-frequency fluctuations, func- tional connectivity density/strength) remains an important question for future work.

The results of our study indicate that $\mathrm{rCBF}$ may be a useful diagnostic marker for distinguishing myelopathyrelated impairments of the $\mathrm{SMC}$, and the $\mathrm{CBF}$ values in the left PCL, the right PCL/SMA, and the right PoCG may help accurately discriminate the CSM from HCs with moderate sensitivity and specificity cut-off points. Furthermore, this method measuring $\mathrm{CBF}$ might provide a straightforward 
view for central nervous system responding of perfusion in postoperative evaluation of decompression in CSM patients.

While our results are interesting and encouraging, several issues need to be further addressed. First, this study was focused on alterations in perfusion of the SMC obtained from healthy subjects using the group ICA toolbox (http:// mialab.mrn.org/data/index.html). Although other alterations in rCBF may exist in undiscovered regions, using a different SMC mask should not change the overall results reported in this study because the calculations of $\mathrm{rCBF}$ are independent of the image mask. Second, decreased CBF of the PMv/ PrCO occurred only on the left side, while increased $\mathrm{CBF}$ of the PoCG occurred only on the right side. Asymmetrical and heterogenic ultrastructural pathological changes may explain this phenomenon, but this needs to be confirmed in the future. Furthermore, our study incorporated a relatively small sample size, and thus, larger samples or additional classifications should be included in future studies. Finally, regarding the physiological basis of $\mathrm{CBF}$ and its association with structural damage at the spinal cord, alterations in blood flow due to single- or multi-level compression of cervical arteries may be one reason for the observed differences, and future work will need to consider blood flow alterations.

In summary, reduced $\mathrm{CBF}$ was observed in operculumintegrated (PMv/PrCO) and motor control (dACC) regions in patients with CSM, but CBF was increased in sensory (PoCG) and motor-sensory (PCL/SMA) regions. These findings are consistent with defects in movement precision, decreased finger dexterity, and the loss of motor skills in CSM patients.

\section{Acknowledgments}

This work was supported, in part, by the National Natural Science Foundation of China (grant numbers 81460329 and 81560284), the Natural Science Foundation of Jiangxi, China (grant number 20161BAB205245), and the Distinguished Young Scholars of Jiangxi Province (grant number 20171BCB23089). The authors are very grateful to the participants for taking part in the present study, and would also like to thank Professor Ze Wang for his help in this ASL study. The funders had no role in the study design, data collection and analysis, decision to publish, or preparation of the manuscript.

\section{Disclosure}

The authors report no conflicts of interest in this work.

\section{References}

1. Zhou FQ, Tan YM, Wu L, Zhuang Y, He LC, Gong HH. Intrinsic functional plasticity of the sensory-motor network in patients with cervical spondylotic myelopathy. Sci Rep. 2015;5:9975.
2. Wen CY, Cui JL, Liu HS, Mak KC, Cheung WY, Luk KD, Hu Y. Is diffusion anisotropy a biomarker for disease severity and surgical prognosis of cervical spondylotic myelopathy? Radiology. 2014;270(1):197-204.

3. Ellingson BM, Salamon N, Holly LT. Advances in MR imaging for cervical spondylotic myelopathy. Eur Spine J. 2015;24 Suppl 2:197-208.

4. Yarbrough CK, Murphy RK, Ray WZ, Stewart TJ. The natural history and clinical presentation of cervical spondylotic myelopathy. $A d v$ Orthop. 2012;2012:480643.

5. Aleksanderek I, McGregor SM, Stevens TK, Goncalves S, Bartha R, Duggal N. Cervical spondylotic myelopathy: metabolite changes in the primary motor cortex after surgery. Radiology. 2017;282(3):817-825.

6. Holly LT, Dong Y, Albistegui-Dubois R, Marehbian J, Dobkin B. Cortical reorganization in patients with cervical spondylotic myelopathy. J Neurosurg Spine. 2007;6(6):544-551.

7. Dong Y, Holly LT, Albistegui-Dubois R, et al. Compensatory cerebral adaptations before and evolving changes after surgical decompression in cervical spondylotic myelopathy. J Neurosurg Spine. 2008;9(6): 538-551.

8. Duggal N, Rabin D, Bartha R, Barry RL, Gati JS, Kowalczyk I, Fink M. Brain reorganization in patients with spinal cord compression evaluated using fMRI. Neurology. 2010;74(13):1048-1054.

9. Tam S, Barry RL, Bartha R, Duggal N. Changes in functional magnetic resonance imaging cortical activation after decompression of cervical spondylosis: case report. Neurosurgery. 2010;67(3):E863-864; discussion E864.

10. Goncalves S, Stevens TK, Doyle-Pettypiece P, Bartha R, Duggal N. $\mathrm{N}$-acetylaspartate in the motor and sensory cortices following functional recovery after surgery for cervical spondylotic myelopathy. J Neurosurg Spine. 2016;25(4):436-443.

11. Craciunas SC, Gorgan MR, Ianosi B, Lee P, Burris J, Cirstea CM. Remote motor system metabolic profile and surgery outcome in cervical spondylotic myelopathy. J Neurosurg Spine. 2017;26(6): 668-678.

12. Zhou F, Gong H, Liu X, Wu L, Luk KD, Hu Y. Increased low-frequency oscillation amplitude of sensorimotor cortex associated with the severity of structural impairment in cervical myelopathy. PloS One. 2014;9(8):e104442.

13. Tan Y, Zhou F, Wu L, Liu Z, Zeng X, Gong H, He L. Alteration of regional homogeneity within the sensorimotor network after spinal cord decompression in cervical spondylotic myelopathy: a resting-state fMRI study. Biomed Res Int. 2015;2015:647958.

14. Zhou F, Wu L, Liu X, Gong H, Luk KD, Hu Y. Characterizing thalamocortical disturbances in cervical spondylotic myelopathy: revealed by functional connectivity under two slow frequency bands. PloS One. 2015;10(6):e0125913.

15. Liang X, Zou Q, He Y, Yang Y. Coupling of functional connectivity and regional cerebral blood flow reveals a physiological basis for network hubs of the human brain. Proc Natl Acad Sci U S A. 2013;110(5):1929-1934.

16. Paulson OB, Hasselbalch SG, Rostrup E, Knudsen GM, Pelligrino D. Cerebral blood flow response to functional activation. J Cereb Blood Flow Metab. 2010;30(1):2-14.

17. Wakasugi N, Kanazawa M, Yajima R, Katsumi K, Endo N, Nishizawa M. A patient with cervical spondylotic myelopathy with $18 \mathrm{~F}-\mathrm{FDG}$ uptake on positron emission tomography/computed tomography. Brain Nerve. 2016;68(2):191-193.

18. Floeth FW, Stoffels G, Herdmann J, Jansen P, Meyer W, Steiger HJ, Langen KJ. Regional impairment of 18 F-FDG uptake in the cervical spinal cord in patients with monosegmental chronic cervical myelopathy. Eur Radiol. 2010;20(12):2925-2932.

19. Borogovac A, Asllani I. Arterial spin labeling (ASL) fMRI: advantages, theoretical constrains, and experimental challenges in neurosciences. Int J Biomed Imaging. 2012;2012:818456.

20. Guo L, Zhang Q, Ding L, et al. Pseudo-continuous arterial spin labeling quantifies cerebral blood flow in patients with acute ischemic stroke and chronic lacunar stroke. Clin Neurol Neurosurg. 2014;125: 229-236. 
21. Xu G, Rowley HA, Wu G, et al. Reliability and precision of pseudocontinuous arterial spin labeling perfusion MRI on 3.0 T and comparison with 15O-water PET in elderly subjects at risk for Alzheimer's disease. NMR Biomed. 2010;23(3):286-293.

22. Ota M, Ishikawa M, Sato N, et al. Pseudo-continuous arterial spin labeling MRI study of schizophrenic patients. Schizophr Res. 2014;154(1-3): 113-118.

23. Goto M, Abe O, Aoki S, et al. Diffeomorphic anatomical registration through exponentiated lie algebra provides reduced effect of scanner for cortex volumetry with atlas-based method in healthy subjects. Neuroradiology. 2013;55(7):869-875.

24. Higo T, Mars RB, Boorman ED, Buch ER, Rushworth MF. Distributed and causal influence of frontal operculum in task control. Proc Natl Acad Sci U S A. 2011;108(110):4230-4235.

25. Ehrsson HH, Spence C, Passingham RE. That's my hand! Activity in premotor cortex reflects feeling of ownership of a limb. Science. 2004;305(5685): 875-877.

26. Bekrater-Bodmann R, Foell J, Kamping S. The importance of ventral premotor cortex for body ownership processing. J Neurosci. 2011;31(26): 9443-9444.

27. Binkofski F, Buccino G. The role of ventral premotor cortex in action execution and action understanding. J Physiol Paris. 2006;99(4-6):396-405.

28. Asemi A, Ramaseshan K, Burgess A, Diwadkar VA, Bressler SL. Dorsal anterior cingulate cortex modulates supplementary motor area in coordinated unimanual motor behavior. Front Hum Neurosci. 2015;9:309.
29. Diwadkar VA, Asemi A, Burgess A, Chowdury A, Bressler SL. Potentiation of motor sub-networks for motor control but not working memory: Interaction of $\mathrm{dACC}$ and SMA revealed by resting-state directed functional connectivity. PLoS One. 2017;12(3):e0172531.

30. Bush G, Vogt BA, Holmes J, Dale AM, Greve D, Jenike MA, Rosen BR. Dorsal anterior cingulate cortex: a role in reward-based decision making. Proc Natl Acad Sci U S A. 2002;99(1):523-528.

31. Hoffstaedter F, Grefkes C, Caspers S, et al. The role of anterior midcingulate cortex in cognitive motor control: evidence from functional connectivity analyses. Hum Brain Mapp. 2014;35(6):2741-2753.

32. Cermik TF, Tuna H, Kaya M, et al. Assessment of regional blood flow in cerebral motor and sensory areas in patients with spinal cord injury. Brain Res. 2006;1109(1):54-59.

33. Baron EM, Young WF. Cervical spondylotic myelopathy: a brief review of its pathophysiology, clinical course, and diagnosis. Neurosurgery. 2007;60(1 Supp1 1):S35-S41.

34. Spasojević G, Malobabic S, Pilipović-Spasojević O, Djukić-Macut $\mathrm{N}$, Maliković A. Morphology and digitally aided morphometry of the human paracentral lobule. Folia Morphol (Warsz). 2013;72(1):10-16.

35. Kowalczyk I, Duggal N, Bartha R. Proton magnetic resonance spectroscopy of the motor cortex in cervical myelopathy. Brain. 2012;135(Pt 2): $461-468$. 


\section{Supplementary material}

Table SI Significantly altered rCBF values in the ROC analysis for differentiating CSM patients from healthy subjects

\begin{tabular}{|c|c|c|c|c|}
\hline Altered rCBF in the CSM patients & Area under the curve & Cut-off point & Sensitivity & Specificity \\
\hline CBF values in the left $\mathrm{PMv} / \mathrm{PrCO}$ & 0.328 & & & \\
\hline$C B F$ values in the bilateral $\mathrm{dACC}$ & 0.398 & & & \\
\hline$C B F$ values in the left $P C L$ & 0.738 & $20.76^{\mathrm{a}}$ & $88.5 \%(16 / 18)$ & $61.1 \%(11 / 18)$ \\
\hline CBF values in the right $P C L / S M A$ & 0.754 & 23.35 & $83.3 \%(15 / 18)$ & $66.7 \%(12 / 18)$ \\
\hline$C B F$ values in the right PoCG & 0.590 & 47.53 & $88.5 \%(26 / 18)$ & $44.4 \%(8 / 18)$ \\
\hline
\end{tabular}

Notes: asing this cut-off point, the $\mathrm{rCBF}$ value of PCL correctly classified 16 of 18 CSM patients and II of $18 \mathrm{HC}$ subjects, resulting in a sensitivity of $88.5 \%$ and a specificity of $61.1 \%$. The means for other cut-off points were similar.

Abbreviations: $\mathrm{dACC}$, dorsal anterior cingulate cortex; HC, healthy control; PCL, paracentral lobule; PMv/ PrCO, premotor ventral/precentral operculum; PoCG, postcentral gyrus; rCBF, regional cerebral blood flow; SMA, supplementary motor area.

The Journal of Pain Research is an international, peer reviewed, open access, online journal that welcomes laboratory and clinical findings in the fields of pain research and the prevention and management of pain. Original research, reviews, symposium reports, hypothesis formation and commentaries are all considered for publication.
Dovepress

The manuscript management system is completely online and includes a very quick and fair peer-review system, which is all easy to use. Visit http://www.dovepress.com/testimonials.php to read real quotes from published authors. 